\title{
Energy-momentum powered gravity and cosmic acceleration
}

\section{Özgür Akarsu}

Department of Physics, Istanbul Technical University, Maslak 34469 Istanbul, Turkey

E-mail: akarsuo@itu.edu.tr

\section{Nihan Katırcı*}

Department of Physics, Istanbul Technical University, Maslak 34469 Istanbul, Turkey

E-mail: nihan.katirci@itu.edu.tr

\section{Suresh Kumar}

Department of Mathematics, BITS Pilani, Pilani Campus, Rajasthan-333031, India

E-mail: suresh.kumar@pilani.bits-pilani.ac.in

We present a summary of the findings in energy-momentum powered gravity (EMPG) model studied in Ö. Akarsu, N. Katırc1, S. Kumar, Phys. Rev. D 97 (2018) 024011. In contrast to theories of non-linear gravity, which are based on generalizing the Einstein-Hilbert curvature contribution to the Lagrangian, we consider non-linear contributions of the usual energy-momentum tensor to the matter Lagrangian. We consider a particular form of them as $f\left(T_{\mu v} T^{\mu v}\right)$ as $f\left(T_{\mu v} T^{\mu v}\right)=$ $\alpha\left(T_{\mu \nu} T^{\mu v}\right)^{\eta}$, where $\alpha$ and $\eta$ are real constants, dubbed as energy-momentum powered gravity (EMPG). We look for viable cosmologies arising from EMPG in the context of the late-time accelerated expansion of the Universe at the background level. We obtain late time acceleration in a dust-dominated Universe without invoking dark energy source like cosmological constant. With a recent compilation of 28 Hubble parameter measurements, we constrain the parameters of the EMPG model and we conclude that, although the underlying physics in the EMPG model is entirely different in the sense that the energy in the EMPG Universe is sourced by pressureless matter only, this model describes an evolution of the Universe similar to that in the $\Lambda$ CDM model.

Corfu Summer Institute 2017 'School and Workshops on Elementary Particle Physics and Gravity' 2-28 September 2017

Corfu, Greece

\footnotetext{
* Speaker.
} 


\section{Introduction}

Expansion of the Universe was expected to be decelerating within general relativity (GR) given that the current Universe is filled with dust. It turned out however that our Universe is in fact expanding with an accelerating rate, which is still a mystery. The present most successful cosmological model capable of describing observed expansion history of the Universe so far is the six-parameter base $\Lambda \mathrm{CDM}$ model that is simple and in reasonably good agreement with the high-precision cosmological data $[1,2,3]$. The cosmological constant $(\Lambda)$ is introduced into GR $[4,5,6,7]$, whose value from observations and quantum field theory calculations as a possible energy density of the quantum vacuum energy are inconsistent with a ratio $\rho_{\Lambda}^{\text {(observation) }} / \rho_{\Lambda}^{\text {(theory) }} \sim 10^{-120}$ $[8,4,9,5,10,11]$. Besides there are some observations that suggest small deviations from/tensions with $\Lambda \mathrm{CDM}[2,3,12]$. Additionally, some tensions between different cosmological data sets may be signalling an incompleteness of $\Lambda \mathrm{CDM}$. There are some parametrizations which allow to probe departure from standard paradigm such as wCDM model and CPL parametrization [13, 14], but these are phenomenological.

On the other hand, standard GR including evolving scalar fields, bulk viscous fluids, generalized Chaplygin gas have been proposed to drive the late time acceleration, usually dubbed as "dark energy". In this regard, modified theories of gravities have also been studied such as the scalartensor theories, Brans-Dicke theories of gravity, $f(R)$ etc. $[15,16,17,18]$. These latter studies focus on, rather than imposing an unknown kind of source, generalising the gravitational Lagrangian from the linear function of the spacetime curvature. However, non-linear function of matter source has remained out of the attention so far, though such higher-order matter terms naturally arise as correction terms in loop quantum gravity [19, 20], and also arise in the brane world models [21]. Energy-momentum tensor (EMT)-type of modifications have started to attract an increasing attention in the recent literature. With some specific choices of the function, $f\left(R, \mathscr{L}_{\mathrm{m}}\right)$, and $f(R, T)$ gravity models have been proposed and studied in Refs. [22, 23].

Energy-momentum powered gravity (EMPG) has been proposed in [24, 25] with a function of $f\left(T_{\mu v} T^{\mu v}\right)=\alpha\left(T_{\mu \nu} T^{\mu v}\right)^{\eta}$, in fact a particular case of the $f\left(R, T_{\mu \nu} T^{\mu v}\right)$ model was proposed in [26]. In this work, we investigate the ranges of the EMPG model parameters $(\alpha, \eta)$, where $\alpha$ and $\eta$ are real constants, for viable cosmologies leading to the late-time acceleration of the Universe with dust only, while keeping the successes of the standard GR at early times. EMPG is an energy density scale dependent model such that $\alpha\left(T_{\mu v} T^{\mu v}\right)^{\eta}$ modification shows its effects at high/low energy densities for $\eta$ values higher/lower than $\frac{1}{2}$, albeit we study low energy density modification to explain late time acceleration. We here demonstrate that only the dust content is sufficient to explain the observed cosmic acceleration. Energy-momentum squared gravity ( $\eta=1$ case) has also been studied in [27, 25], $\alpha T_{\mu v} T^{\mu v}$ modification ( $\rho^{2}$ terms appear next to the standard $\rho$ term) manifests itself at larger values of $\rho$, i.e., relatively early Universe such as Big-bang nucleosynthesis (BBN) processes, leaving the late-time Universe unaltered. It is obvious that it is not possible to use EMSG modification at late times to drive accelerated expansion, hence $\Lambda$ should be considered in the EMSG framework. In EMSG, it is important to understand the high energy density scales where this theory would deviate from GR considerably, and hence the strongest constraints can be obtained from neutron stars. In [28], authors discuss what contributions EMSG can provide to the physics of neutron stars, in particular, in relevance with the so called hyperon puzzle in neutron 
stars.

\section{Energy-Momentum Powered Gravity}

In EMPG, EMT-powered form, $f\left(T_{\mu v} T^{\mu v}\right)=\alpha\left(T_{\mu v} T^{\mu v}\right)^{\eta}$ is added to Einstein-Hilbert (EH) action as follows:

$$
S=\int\left[\frac{1}{2 \kappa} R+\alpha\left(T_{\mu v} T^{\mu v}\right)^{\eta}+\mathscr{L}_{\mathrm{m}}\right] \sqrt{-g} \mathrm{~d}^{4} x
$$

where $g$ is the determinant of the metric, $\kappa$ is Newton's gravitational constant, $R$ is the curvature scalar, and $\mathscr{L}_{\mathrm{m}}$ is the matter Lagrangian density. ${ }^{1}$ Here $\eta$ is the power of the scalar from selfcontraction of the EMT, and $\alpha$ is a constant that would contributes to the coupling strength of the EMT-powered modification to gravity. The Einstein field equations and the covariant divergence of the EMT become

$$
\begin{gathered}
G_{\mu v}=\kappa T_{\mu v}+\kappa \alpha\left(T_{\sigma \varepsilon} T^{\sigma \varepsilon}\right)^{\eta}\left[g_{\mu \nu}-2 \eta \frac{\theta_{\mu v}}{T_{\sigma \varepsilon} T^{\sigma \varepsilon}}\right] \\
\nabla^{\mu} T_{\mu v}=-\alpha g_{\mu v} \nabla^{\mu}\left(T_{\sigma \varepsilon} T^{\sigma \varepsilon}\right)^{\eta}+2 \alpha \eta \nabla^{\mu}\left(\frac{\theta_{\mu v}}{\left(T_{\sigma \varepsilon} T^{\sigma \varepsilon}\right)^{1-\eta}}\right) .
\end{gathered}
$$

where $\theta_{\mu \nu}$ is the new tensor defined as

$$
\theta_{\mu v}=T^{\sigma \varepsilon} \frac{\delta T_{\sigma \varepsilon}}{\delta g^{\mu \nu}}+T_{\sigma \varepsilon} \frac{\delta T^{\sigma \varepsilon}}{\delta g^{\mu \nu}}=-2 \mathscr{L}_{\mathrm{m}}\left(T_{\mu \nu}-\frac{1}{2} g_{\mu \nu} T\right)-T T_{\mu \nu}+2 T_{\mu}^{\gamma} T_{v \gamma}
$$

In the context of cosmology, we study EMPG considering the spatially maximally symmetric spacetime metric, i.e., the Robertson-Walker metric, with flat space-like sections

$$
d s^{2}=-d t^{2}+a^{2}\left(\mathrm{~d} x^{2}+\mathrm{d} y^{2}+\mathrm{d} z^{2}\right)
$$

where the scale factor $a=a(t)$ is a function of cosmic time $t$ only, and the perfect fluid form of the EMT is given by

$$
T_{\mu v}=(\rho+p) u_{\mu} u_{v}+p g_{\mu v}
$$

where $\rho$ is the energy density, $p$ is the pressure, and $u_{\mu}$ is the four-velocity satisfying the $u_{\mu} u^{\mu}=-1$ and $\nabla_{v} u^{\mu} u_{\mu}=0$ conditions. We describe the physical ingredient of the Universe with a barotropic equation-of-state (EoS) as $\frac{p}{\rho}=w=$ const. and using (2.7), we obtain

$$
\theta_{\mu v}=-\rho^{2}(3 w+1)(w+1) u_{\mu} u_{v} \quad \text { and } \quad T_{\mu v} T^{\mu v}=\rho^{2}\left(3 w^{2}+1\right),
$$

respectively. Using Eqs. (2.8) with background metric (2.6) in the field equations (2.3), we obtain the following set of two linearly independent differential equations with two unknown functions $H$

${ }^{1}$ We define EMT as

$$
T_{\mu \nu}=-\frac{2}{\sqrt{-g}} \frac{\delta\left(\sqrt{-g} \mathscr{L}_{\mathrm{m}}\right)}{\delta g^{\mu v}}=g_{\mu v} \mathscr{L}_{\mathrm{m}}-2 \frac{\partial \mathscr{L}_{\mathrm{m}}}{\partial g^{\mu \nu}},
$$

which depends only on the metric tensor components, and not on its derivatives and we choose $\mathscr{L}_{\mathrm{m}}=p$. 
and $\rho$ :

$$
\begin{aligned}
3 H^{2} & =\kappa \rho+\kappa^{\prime} \rho_{0}\left(\frac{\rho}{\rho_{0}}\right)^{2 \eta}, \\
-2 \dot{H}-3 H^{2} & =\kappa w \rho+\frac{\kappa^{\prime} \rho_{0}}{2 \eta-1+\frac{8 w \eta}{3 w^{2}+1}}\left(\frac{\rho}{\rho_{0}}\right)^{2 \eta},
\end{aligned}
$$

where $H=\dot{a} / a$ is the Hubble parameter and the subscript ${ }_{0}$ denotes the present-day values of the parameters. The constant $\kappa^{\prime}$ is the gravitational coupling constant of the EMT-powered modification, and is given by

$$
\kappa^{\prime}=\alpha^{\prime} \kappa=\alpha \kappa \rho_{0}^{2 \eta-1}\left(3 w^{2}+1\right)^{\eta}\left[2 \eta-1+\frac{8 w \eta}{3 w^{2}+1}\right]
$$

here, the terms that appear in the field equations due to the EMT-powered term in the action couple to gravity with a different strength as $\kappa^{\prime}=\alpha^{\prime} \kappa$, where $\alpha^{\prime}$ is the ratio of $\kappa^{\prime}$ with respect to the standard Newtonian coupling $\kappa$. We should here note that, provided that $\eta \neq 0, \alpha^{\prime}=\alpha^{\prime}\left(\alpha, \eta, \rho_{0}, w\right)$ depends not only on $\alpha$ but also on the energy density $\rho_{0}$ today and EoS parameter of the source $w$. This implies that the EMT-powered term would lead in general to a violation of the equivalence principle, intimately connected with some of the basic aspects of attempts at unifying gravity with particle physics such as string theories (see Ref. [29] and references therein). We further note that the first Friedmann equation (2.13) is in the form of the well-known Cardassian expansion $\left(H^{2}=A \rho+B \rho^{n}\right.$, with $A, B$, and $n$ being constants) [30] where $\rho^{n}$ can generically appear in braneworld scenarios $[30,31]$. On the other hand, in the second Friedmann equation (2.14) is in the form of the pressure of the generalized Chaplygin gas ( $p=-A / \rho^{\alpha}$, where $A$ is a positive constant) [32,33]. But in EMPG, we see that the additional pressure term which appears due to the EMT-powered modification. Additionally, for the $\eta=1$ case (EMSG), the total pressure [the right-hand side of Eq. (2.14)] is similar to the quadratic equation of state ( $p=p_{0}+\alpha \rho+\beta \rho^{2}$, where $p_{0}, \alpha$, and $\beta$ are constants) of dark energy [34]. However, one may check that our model in fact does not correspond to any of them, namely, the modified Friedmann equations of our model (2.13) and (2.14) do not simultaneously match the Friedmann equations of each of these models. In these models, energy-momentum tensor is conserved whereas the local/covariant energy conservation is violated in our model given in (2.4) as

$$
\dot{\rho}+3 H(1+w) \rho=-2 \alpha^{\prime} \eta\left[\dot{\rho}+3 H \rho\left(\frac{1+\frac{4 w}{3 w^{2}+1}}{2 \eta-1+\frac{8 w \eta}{3 w^{2}+1}}\right)\right]\left(\frac{\rho}{\rho_{0}}\right)^{2 \eta-1} .
$$

Here we can see that the local/covariant energy-momentum conservation $\nabla^{\mu} T_{\mu \nu}=0$, which would lead to $\rho \propto a^{-3(1+w)}$, is not satisfied for $\alpha^{\prime} \neq 0$ in general.

\subsection{Dust only Universe in EMPG}

We consider here in the presence of only a pressureless fluid using (2.9)-(2.10) with $w=0$, we obtain

$$
\begin{gathered}
3 H^{2}=\kappa \rho_{\mathrm{m}}+\kappa^{\prime} \rho_{\mathrm{m}, 0}\left(\frac{\rho_{\mathrm{m}}}{\rho_{\mathrm{m}, 0}}\right)^{2 \eta}, \\
-2 \dot{H}-3 H^{2}=\frac{\kappa^{\prime} \rho_{\mathrm{m}, 0}}{2 \eta-1}\left(\frac{\rho_{\mathrm{m}}}{\rho_{\mathrm{m}, 0}}\right)^{2 \eta},
\end{gathered}
$$


where $\kappa^{\prime}=\alpha^{\prime} \kappa=\alpha \kappa(2 \eta-1) \rho_{0}^{2 \eta-1}$ is the gravitational coupling constant of the EMT-powered modification. In this work, we focus on the late time dynamics of Universe at background level in the presence of dust where the radiation is negligible.

\subsection{Late-time acceleration}

The late-time acceleration of the Universe takes place at relatively low energies, and hence the EMPG modification should be effective at sufficiently low energy densities. The modification should be negligible at high energy densities, namely, at energies higher than that of recombination. In this way, the successes of the standard cosmology would be untouched. Before observational analysis, suitable ranges of the model parameters $\alpha^{\prime}$ and $\eta$ can be discussed in theoretical grounds. Knowing that the energy density of the matter source $\rho$ should be positive, we would like to ensure that the EMT-powered contribution to the Hubble parameter [the latter term in Eq. (2.13)] is positive as well. Hence we consider $\kappa^{\prime}>0$ which corresponds to $\alpha^{\prime}>0$. For $\eta=0$ case in EMPG, we obtain $3 H^{2}=\kappa \rho_{\mathrm{m}, 0}\left(\frac{a}{a_{0}}\right)^{-3}+\kappa^{\prime} \rho_{\mathrm{m}, 0}$, hence, the model yields the same mathematical structure as the $\Lambda \mathrm{CDM}$ model $3 H^{2}=\kappa \rho_{\mathrm{m}, 0}\left(\frac{a}{a_{0}}\right)^{-3}+\kappa \rho_{\Lambda}$, where $\rho_{\Lambda}=$ const. but we should stress that the underlying physics of these two models are completely different. For instance, if we consider an empty Universe $\left(\rho_{\mathrm{m}, 0}=0\right)$, we find $H=0$ (a static Universe) in our model, whereas we find $H=\sqrt{\frac{\rho_{\Lambda}}{3}}=$ const. (the de Sitter solution) in the $\Lambda$ CDM model. For today $a=a_{0}$, we obtain $3 H_{0}^{2}=\left(\kappa+\kappa^{\prime}\right) \rho_{\mathrm{m}, 0}$ for our model, and $3 H_{0}^{2}=\kappa\left(\rho_{\mathrm{m}, 0}+\rho_{\Lambda}\right)$ for the $\Lambda$ CDM model, the two models for $\alpha^{\prime}=\rho_{\Lambda} / \rho_{\mathrm{m}, 0}=\Omega_{\Lambda} / \Omega_{\mathrm{m}, 0}$ become the same. From the recent Planck results [3] giving $\Omega_{\Lambda, 0} \sim 0.69$ for the current Universe, we can estimate that $\alpha^{\prime} \sim 2.2$. Depending on the EMTpowered modification, pressureless matter itself mimics the cosmological constant while preserving the standard dust contribution via the usual EMT term.

Even $\eta=0$ case in EMPG has some promising features comparing to $\Lambda \mathrm{CDM}$ such as alleviating cosmological constant problem (for details, see [24]), we elaborate on $\eta \neq 0$ cases in EMPG, and explore the range of the values of $\eta$ that give accelerated expansion at late times. We aim that EMT-powered terms manifest at lower values of $\rho$-relatively late Universe-, hence we should consider $\eta<\frac{1}{2}$ cases and check that deceleration parameter using Eqs.(2.13)-(2.14):

$$
q=-1-\frac{\dot{H}}{H^{2}}=\frac{1+2 \alpha^{\prime}\left(\frac{\eta+1}{2 \eta-1}\right)\left(\frac{\rho_{\mathrm{m}}}{\rho_{\mathrm{m}, 0}}\right)^{2 \eta-1}}{2+2 \alpha^{\prime}\left(\frac{\rho_{\mathrm{m}}}{\rho_{\mathrm{m}, 0}}\right)^{2 \eta-1}}
$$

and we investigate the evolution the Universe at $z \lesssim 1100$ as well as the late-time accelerated expansion, which starts at $z \sim 0.6$. Because the condition $\alpha^{\prime}>0$ makes the denominator always positive, $q$ can take negative values if

$$
-1<\eta<\frac{1}{2}
$$

due to the term $\frac{\eta+1}{2 \eta-1}$ in the numerator. We note that the upper limit coincides with the one we obtained in order for the EMPG modification to be effective at lower energy density values. Thus, the condition (2.16) guarantees standard GR at earlier times, and cosmic acceleration at later times by means of the EMPG modification. Finally, we obtain accelerated expansion in the present 
Universe at $z=0$, implying $\rho_{\mathrm{m}}=\rho_{\mathrm{m}, 0}$, provided that

$$
-1+\frac{3}{2 \alpha^{\prime}+2}<\eta<\frac{1}{2}
$$

which is a stronger condition than the one given in Eq. (2.16) for all positive values of $\alpha^{\prime}$. We next see from Eq. (2.15) that $q \approx \frac{\eta+1}{2 \eta-1}$ for $\rho_{\mathrm{m}} \ll \rho_{\mathrm{m}, 0}$ provided that $\rho_{\mathrm{m}}$ can decrease to sufficiently small values under the condition (2.16).

Using Eqs. (2.8) and the metric (2.6), the energy conservation equation (2.4) for pressureless matter $(w=0)$ reads

$$
\frac{\dot{\rho}_{\mathrm{m}}}{\rho_{\mathrm{m}}}=-3 H \frac{1+\frac{2 \alpha^{\prime} \eta}{2 \eta-1}\left(\frac{\rho_{\mathrm{m}}}{\rho_{\mathrm{m}, 0}}\right)^{2 \eta-1}}{1+2 \alpha^{\prime} \eta\left(\frac{\rho_{\mathrm{m}}}{\rho_{\mathrm{m}, 0}}\right)^{2 \eta-1}}
$$

whose solution gives

$$
\frac{\rho_{\mathrm{m}}}{\rho_{\mathrm{m}, 0}}\left[\frac{1+\frac{2 \eta \alpha^{\prime}}{2 \eta-1}\left(\frac{\rho_{\mathrm{m}}}{\rho_{\mathrm{m}, 0}}\right)^{2 \eta-1}}{1+\frac{2 \eta \alpha^{\prime}}{2 \eta-1}}\right]^{\frac{2 \eta-2}{2 \eta-1}}=a^{-3} .
$$

It should be noted here that we are not able to give an explicit solution for $\rho_{\mathrm{m}}(a)$ since it is not possible to isolate $\rho_{\mathrm{m}}$ except for a couple of particular cases of $\eta$ in this equation and we find that the following explicit expression for the matter energy density $\rho_{\mathrm{m}}$ in terms of $z$ is a very good approximation [with the maximum relative error $\left(\delta \rho_{\mathrm{m}} / \rho_{\mathrm{m}}\right)$ on the order of $10^{-5}$ ] to Eq. (2.19) for $\rho_{\mathrm{m}} / \rho_{\mathrm{m}, 0} \gtrsim 1$ and $\eta \sim 0$

$$
\rho_{\mathrm{m}}=\rho_{\mathrm{m}, 0}\left[\beta(1+z)^{3}+1-\beta\right]
$$

where $\beta=\left(1+\frac{2 \eta \alpha^{\prime}}{2 \eta-1}\right)^{\frac{2 \eta-2}{2 \eta-1}}$.

\subsection{Observational constraints}

Substituting Eq. (2.20) and the Hubble constant $H_{0}^{2}=\frac{\kappa}{3}\left(1+\alpha^{\prime}\right) \rho_{\mathrm{m}, 0}$ into Eq. (2.13), the approximated modified Friedmann equation in terms of redshift $z$ is

$$
\frac{H^{2}}{H_{0}^{2}}=\frac{1}{1+\alpha^{\prime}}\left[\beta(1+z)^{3}+1-\beta\right]+\frac{\alpha^{\prime}}{1+\alpha^{\prime}}\left[\beta(1+z)^{3}+1-\beta\right]^{2 \eta}
$$

subject to the conditions $\rho_{\mathrm{m}} \gtrsim \rho_{\mathrm{m}, 0}>\rho_{\mathrm{m}, \min }$ (or $z \gtrsim 0$ ) and the ones given in Eqs. (2.16). Now we can constrain the EMPG model parameters $\eta, \alpha^{\prime}$ and $H_{0}$ with the observational data and we use the compilation of 28 Hubble parameter measurements spanning the redshift range $0.07 \leq z \leq 2.3$. The $28 H(z)$ data points were compiled in Ref. [35] to determine constraints on the parameters of various dark energy models. ${ }^{2}$ Our results are presented in Table 1.

${ }^{2}$ We constrain parameters $\left(H_{0}, \alpha^{\prime}, \eta\right)$ of the EMPG model by minimizing

$$
\chi_{H}^{2}\left(H_{0}, \alpha^{\prime}, \eta\right)=\sum_{i=1}^{28} \frac{\left[H^{\mathrm{th}}\left(z_{i} ; H_{0}, \alpha^{\prime}, \eta\right)-H^{\mathrm{obs}}\left(z_{i}\right)\right]^{2}}{\sigma_{\mathrm{H}, i}^{2}}
$$

for 28 measured $H^{\mathrm{obs}}\left(z_{i}\right)$ 's with variance $\sigma_{\mathrm{H}, i}^{2}$ at redshift $z_{i}$ whereas $H^{\text {th }}$ is the predicted value of $H(z)$ in the EMPG model. The parameter space $\left(H_{0}, \alpha^{\prime}, \eta\right)$ of the model is explored by using the Markov chain Monte Carlo method coded in the publicly available package COSMOMC [36]. 


\begin{tabular}{cccc}
\hline \hline Parameter & Mean & $68 \%$ C.L. & $95 \%$ C.L. \\
\hline$H_{0}$ & 68.7 & {$[66.5,70.9]$} & {$[64.2,73.2]$} \\
$\alpha^{\prime}$ & 2.80 & {$[2.54,3.05]$} & {$[2.42,3.17]$} \\
$\eta$ & -0.003 & {$[-0.014,0.009]$} & {$[-0.026,0.020]$} \\
\hline
\end{tabular}

Table 1: Mean values of EMPG model parameters are displayed with $68 \%$ and $95 \%$ confidence levels (C.L.) with $\chi_{\min }^{2}=17$.

We first note that the values of $\alpha^{\prime}$ and $\eta$ satisfy all of the conditions (2.16), (2.17) as described in the previous section for a viable cosmology. We note that the mean value of $\eta$ is almost equal to zero, signaling that our model-in light of observational data-predicts a $\Lambda$ CDM-type background evolution at least up to the present time. The constraints on the $\Lambda$ CDM model parameters read as $H_{0}=68.3_{-2.6-5.1}^{+2.7+5.2} \mathrm{~km} \mathrm{~s}^{-1} \mathrm{Mpc}^{-1}$ and $\Omega_{\mathrm{m}, 0}=0.276_{-0.039-0.068}^{+0.032+0.072}$, with error limits at the $1 \sigma$ and $2 \sigma$ confidence levels and $\chi_{\min }^{2}=17$ (see Table I of Ref. [37]). We conclude that, although the underlying physics in the EMPG model is entirely different in the sense that the energy in the EMPG Universe is sourced by pressureless matter only, this model describes an evolution of the Universe similar to that in the $\Lambda \mathrm{CDM}$ model.

We also obtain the constraint on the parameter $\alpha$ as

$$
\alpha=-0.60_{-0.69}^{+0.50} \times 10^{-8}\left(\mathrm{erg} / \mathrm{cm}^{3}\right)^{1-2 \eta} \quad(95 \% \text { C.L. }) .
$$

As is shown in (2.23), the units of $\alpha$ depend on $\eta$, which indicates that each different value of $\eta$ should be considered as another gravity theory. For relevant details and figures, see [24].

\section{Concluding remarks and future perspectives}

The EMPG is a modified theory of gravitation constructed by the addition of $\alpha\left(T_{\mu \nu} T^{\mu v}\right)^{\eta}$ term to the EH action of GR, we summarize above the relevant findings in the context of the late-time accelerated expansion of the Universe. We have discussed the conditions under which it leads to viable cosmologies, and showed that there are ranges of the parameters of the EMPG model in which it approaches standard GR at high energy densities and the accelerated expansion at sufficiently low energy densities without invoking a cosmological constant or any other dark energy source. In the presence of only a matter source, EMPG can give rise to precisely the same background evolution as the $\Lambda \mathrm{CDM}$ model when $\eta=0$, and when $\eta \sim 0$ model predicts an evolution similar to that of the $w C D M$ model. Although we obtain $\Lambda$ CDM-like model $(\eta=0)$ and $w C D M-l i k e$ $(\eta \sim 0)$ models, the underlying physics of the EMPG-driven cosmologies are entirely different than what we have in the $\Lambda \mathrm{CDM}$ and $w \mathrm{CDM}$ models. Our model alleviates the cosmological constant problem that arises when a cosmological constant is introduced as in the $\Lambda \mathrm{CDM}$ model, and the issue of introducing an ad hoc scalar field that can lead to quintessence and/or phantom (leading additional severe problems) dark energy source with a constant or slightly varying equation-of-state parameter as in the $w \mathrm{CDM}$-like models. We have constrained EMPG model parameters from the 
recent compilation of 28 Hubble parameter measurements reveal that the EMPG model describes an evolution of the Universe similar to that in the $\Lambda \mathrm{CDM}$ model.

The range of possible values for $\eta$ on theoretical grounds is discussed in detail in [24] and As remarked above, $\eta$ determines the order of non-linear contributions of matter term, hence EMPG model can serve as a workable solution for various problems of standard cosmology at very different energy density scales. In EMPG model, the case $\eta>1 / 2$ may be effective at high energy densities; e.g. relevant to early Universe and dense compact astrophysical objects, in line with that in [28], energy-momentum squared gravity (EMSG) - corresponds to $\eta=1$ case of EMPG- has been studied and $\alpha$ parameter has been constrained using neutron stars. Under this astrophysical constraint, cosmological implications of EMSG model have also been discussed. EMSG leaves the most important features of standard cosmology such as the standard big bang nucleosynthesis unaltered. On the other hand studying EMPG model for $\eta>1 / 2$ in the context of BBN would certainly be interesting and these cases may differ from the standard GR and EMSG, even at the very beginning of early Universe it has some different predictions. Namely, it was shown that in [25], the EMPG model for $\eta>1 / 2$ can replace the initial singularity with an initial bounce and avoid spatial anisotropy from dominating the Universe about the initial singularity.

\section{References}

[1] E. Komatsu et al. [WMAP Collaboration], Seven-Year Wilkinson Microwave Anisotropy Probe (WMAP) observations: Cosmological interpretation, Astrophys. J. Suppl. Ser. 192, 18 (2011), [arXiv:1001.4538 [astro-ph.CO]].

[2] E. Aubourg et al., Cosmological implications of baryon acoustic oscillation measurements, Phys. Rev. D 92, 123516 (2015), [arXiv:1411.1074 [astro-ph.CO]].

[3] P. A. R. Ade et al. [Planck Collaboration], Planck 2015 results. XIII. Cosmological parameters, Astron. Astrophys. 594, A13 (2016), [arXiv:1502.01589 [astro-ph.CO]].

[4] V. Sahni and A. A. Starobinsky, The case for a positive cosmological $\Lambda$-term, Int. J. Mod. Phys. D 9 , 373 (2000), [arXiv:astro-ph/9904398].

[5] P. J. E. Peebles and B. Ratra, The cosmological constant and dark energy, Rev. Mod. Phys. 75, 559 (2003), [arXiv:astro-ph/0207347].

[6] E. J. Copeland, M. Sami and S. Tsujikawa, Dynamics of dark energy, Int. J. Mod. Phys. D 15, 1753 (2006), [arXiv:hep-th/0603057].

[7] K. Bamba, S. Capozziello, S. Nojiri and S. D. Odintsov, Dark energy cosmology: The equivalent description via different theoretical models and cosmography tests, Astrophys. Space Sci. 342, 155 (2012), [arXiv:1205.3421 [gr-qc]].

[8] S. Weinberg, The cosmological constant problem, Rev. Mod. Phys. 61, 1 (1989).

[9] S. M. Carroll, The cosmological constant, Living Rev. Relativity 4, 1 (2001), [arXiv:astro-ph/0004075].

[10] V. Sahni, Dark matter and dark energy, Lect. Notes Phys. 653, 141 (2004), [arXiv:astro-ph/0403324].

[11] P. Bull et al., Beyond $\Lambda$ CDM: Problems, solutions, and the road ahead, Phys. Dark Univ. 12, 56 (2016), [arXiv:1512.05356 [astro-ph.CO]]. 
[12] G. B. Zhao et al., Dynamical dark energy in light of the latest observations, Nature Astron. 1, 627 (2017), [arXiv:1701.08165 [astro-ph.CO]].

[13] M. Chevallier and D. Polarski, Accelerating Universes with scaling dark matter, Int. J. Mod. Phys. D 10, 213 (2001), [arXiv:gr-qc/0009008].

[14] E. V. Linder, Exploring the expansion history of the Universe, Phys. Rev. Lett. 90, 091301 (2003), [arXiv:astro-ph/0208512].

[15] A. De Felice and S. Tsujikawa, $f(R)$ theories, Living Rev. Relativity 13, 3 (2010), [arXiv:1002.4928 [gr-qc]].

[16] T. Clifton, P. G. Ferreira, A. Padilla and C. Skordis, Modified gravity and cosmology, Phys. Rep. 513, 1 (2012), [arXiv:1106.2476 [astro-ph.CO]].

[17] S. Capozziello and M. De Laurentis, Extended theories of gravity, Phys. Rep. 509, 167 (2011), [arXiv:1108.6266 [gr-qc]].

[18] S. Nojiri, S. D. Odintsov and V. K. Oikonomou, Modified gravity theories on a nutshell: Inflation, bounce and late-time evolution, Phys. Rep. 692, 1 (2017), [arXiv:1705.11098 [gr-qc]].

[19] A. Ashtekar, T. Pawlowski and P. Singh, Quantum nature of the big bang: Improved dynamics, Phys. Rev. D 74, 084003 (2006), [arXiv:gr-qc/0607039].

[20] A. Ashtekar and P. Singh, “Loop Quantum Cosmology: A Status Report,” Class. Quant. Grav. 28 213001(2011), [arXiv:1108.0893 [gr-qc]].

[21] P. Brax and C. van de Bruck, "Cosmology and brane worlds: A Review," Class. Quant. Grav. 20 R201 (2003), [arXiv:hep-th/0303095].

[22] T. Harko and F. S. N. Lobo, $f\left(R, \mathscr{L}_{\mathrm{m}}\right)$ gravity, Eur. Phys. J. C 70, 373 (2010), [arXiv:1008.4193 [gr-qc]].

[23] T. Harko, F. S. N. Lobo, S. Nojiri and S. D. Odintsov, $f(R, T)$ gravity, Phys. Rev. D 84, 024020 (2011), [arXiv:1104.2669 [gr-qc]].

[24] Ö. Akarsu, N. Katırcı and S. Kumar, Cosmic acceleration in a dust only Universe via energy-momentum powered gravity, Phys. Rev. D 97 2, 024011(2018), [arXiv:1709.02367 [gr-qc]].

[25] C. V. R. Board and J. D. Barrow, Cosmological Models in Energy-Momentum-Squared Gravity, Phys. Rev. D 96 12, 123517 (2017), [arXiv:1709.09501 [gr-qc]].

[26] N. Katırcı and M. Kavuk, $f\left(R, T_{\mu v} T^{\mu v}\right)$ gravity and Cardassian-like expansion as one of its consequences, Eur. Phys. J. Plus 129163 (2014), [arXiv:1302.4300 [gr-qc]].

[27] M. Roshan and F. Shojai, Energy-Momentum Squared Gravity, Phys. Rev. D 94 4, 044002 (2016), [arXiv:1607.06049 [gr-qc]].

[28] Ö. Akarsu, J. D. Barrow, S. Çıkıntoğlu, K. Y. Ekşi and N. Katırcı, Constraint on energy-momentum squared gravity from neutron stars and its cosmological implications, [arXiv:1802.02093 [gr-qc]].

[29] J. P. Uzan, Varying constants, gravitation and cosmology, Living Rev. Relativity 14, 2 (2011), [arXiv:1009.5514 [astro-ph.CO]].

[30] K. Freese and M. Lewis, Cardassian expansion: A model in which the universe is flat, matter dominated, and accelerating, Phys. Lett. B 540, 1 (2002), [arXiv:astro-ph/0201229].

[31] D. J. H. Chung and K. Freese, Cosmological challenges in theories with extra dimensions and remarks on the horizon problem, Phys. Rev. D 61, 023511 (2000), [arXiv:hep-ph/9906542]. 
[32] A. Y. Kamenshchik, U. Moschella and V. Pasquier, An alternative to quintessence, Phys. Lett. B 511, 265 (2001), [arXiv:gr-qc/0103004]

[33] M. C. Bento, O. Bertolami and A. A. Sen, Generalized Chaplygin gas, accelerated expansion and dark energy matter unification, Phys. Rev. D 66, 043507 (2002), [arXiv:gr-qc/0202064].

[34] K. N. Ananda and M. Bruni, Cosmo-dynamics and dark energy with non-linear equation of state: a quadratic model, Phys. Rev. D 74, 023523 (2006), [arXiv:astro-ph/0512224].

[35] O. Farooq and B. Ratra, Hubble parameter measurement constraints on the cosmological deceleration-acceleration transition redshift, Astrophys. J. 766, L7 (2013), [arXiv:1301.5243 [astro-ph.CO]].

[36] A. Lewis and S. Bridle, Cosmological parameters from CMB and other data: A Monte Carlo approach, Phys. Rev. D 66, 103511 (2002), [arXiv:astro-ph/0205436].

[37] Y. Chen, S. Kumar and B. Ratra, Determining the Hubble constant from Hubble parameter measurements, Astrophys. J. 835, 186 (2017), [arXiv:1606.07316 [astro-ph.CO]]. 